\title{
NEW ATLAS9 AND MARCS MODEL ATMOSPHERE GRIDS FOR THE APACHE POINT OBSERVATORY GALACTIC EVOLUTION EXPERIMENT (APOGEE)
}

\author{
Sz. Mészáros ${ }^{1,2}$, C. Allende Prieto ${ }^{1,2}$, B. Edvardsson ${ }^{3}$, F. Castellit ${ }^{4}$, A. E. García Pérez ${ }^{5}$, B. Gustafsson ${ }^{3}$, \\ S. R. Majewski ${ }^{5}$, B. Plez ${ }^{6}$, R. Schiavon ${ }^{7}$, M. Shetrone $^{8}$, and A. De Vicente ${ }^{1,2}$ \\ ${ }^{1}$ Instituto de Astrofísica de Canarias (IAC), E-38200 La Laguna, Tenerife, Spain \\ ${ }^{2}$ Departamento de Astrofísica, Universidad de La Laguna (ULL), E-38206 La Laguna, Tenerife, Spain \\ ${ }^{3}$ Department of Physics and Astronomy, Division of Astronomy and Space Physics, Box 515, SE-751 20 Uppsala, Sweden \\ ${ }^{4}$ Istituto Nazionale di Astrofisica, Osservatorio Astronomico di Trieste, via Tiepolo 11, I-34143 Trieste, Italy \\ ${ }^{5}$ Department of Astronomy, University of Virginia, P.O. Box 400325, Charlottesville, VA 22904-4325, USA \\ ${ }^{6}$ Laboratoire Univers et Particules de Montpellier, Université Montpellier 2, CNRS, F-34095 Montpellier, France \\ ${ }^{7}$ Gemini Observatory, 670 North A'ohoku Place, Hilo, HI 96720, USA \\ ${ }^{8}$ McDonald Observatory, University of Texas, Austin, TX 78712, USA \\ Received 2012 June 11; accepted 2012 August 6; published 2012 September 14
}

\begin{abstract}
We present a new grid of model photospheres for the SDSS-III/APOGEE survey of stellar populations of the Galaxy, calculated using the ATLAS9 and MARCS codes. New opacity distribution functions were generated to calculate ATLAS9 model photospheres. MARCS models were calculated based on opacity sampling techniques. The metallicity $([\mathrm{M} / \mathrm{H}])$ spans from -5 to 1.5 for ATLAS and -2.5 to 0.5 for MARCS models. There are three main differences with respect to previous ATLAS9 model grids: a new corrected $\mathrm{H}_{2} \mathrm{O}$ line list, a wide range of carbon $([\mathrm{C} / \mathrm{M}])$ and $\alpha$ element $[\alpha / \mathrm{M}]$ variations, and solar reference abundances from Asplund et al. The added range of varying carbon and $\alpha$-element abundances also extends the previously calculated MARCS model grids. Altogether, 1980 chemical compositions were used for the ATLAS9 grid and 175 for the MARCS grid. Over 808,000 ATLAS9 models were computed spanning temperatures from $3500 \mathrm{~K}$ to $30,000 \mathrm{~K}$ and $\log g$ from 0 to 5 , where larger temperatures only have high gravities. The MARCS models span from $3500 \mathrm{~K}$ to $5500 \mathrm{~K}$, and $\log g$ from 0 to 5 . All model atmospheres are publicly available online.
\end{abstract}

Key words: astrochemistry - radiative transfer - stars: atmospheres - surveys

Online-only material: color figures

\section{INTRODUCTION}

The Apache Point Observatory Galactic Evolution Experiment (APOGEE; Allende Prieto et al. 2008) is a large-scale, near-infrared, high-resolution spectroscopic survey of Galactic stars, and it is one of the four experiments in the Sloan Digital Sky Survey-III (SDSS-III; Eisenstein et al. 2011; Gunn et al. 2006; Aihara et al. 2011). APOGEE will obtain high signal-tonoise ratio, $R \sim 22,500$ spectra for 100,000 stars in the Milky Way Galaxy, for which accurate chemical abundances, radial velocities, and physical parameters will be determined. APOGEE data will shed new light on the formation of the Milky Way, as well as its chemical and dynamical evolution. To achieve its science goals, APOGEE needs to determine abundances for about 15 elements to an accuracy of 0.1 dex. To attain this precision, a large model photosphere database with up-to-date solar abundances is required. We chose to build the majority of APOGEE's model photosphere database on ATLAS9 and MARCS calculations.

ATLAS (Kurucz 1979) is widely used as a universal LTE onedimensional plane-parallel atmosphere modeling code, which is freely available from Robert Kurucz's Web site. ${ }^{9}$ ATLAS9 (Kurucz 1993) handles the line opacity with the opacity distribution functions (ODF), which greatly simplifies and reduces the computation time (Strom \& Kurucz 1966; Kurucz 2005; Castelli 2005b). ATLAS uses the mixing-length scheme for convective energy transport. It consists of pretabulating the line opacity as a function of temperature and gas pressure in a given number of wavelength intervals that cover the whole wavelength range from far-ultraviolet to far-infrared. For computational reasons,

\footnotetext{
9 http://kurucz.harvard.edu
}

in each interval the line opacities are rearranged according to strength rather than wavelength. For each selected metallicity and microturbulent velocity, an ODF table has to be computed. While the computation of the ODFs is very time consuming, extensive grids of model atmospheres and spectrophotometric energy distributions can be computed in a short time once the required ODF tables are available.

ATLAS12 (Kurucz 2005; Castelli 2005a) uses the opacity sampling (OS) method to calculate the opacity at 30,000 points. The high-resolution synthetic spectrum at a selected resolution can then be obtained by running SYNTHE (Kurucz \& Avrett 1981). More recently, Lester \& Neilson (2008) have developed SATLAS_ODF and SATLAS_OS, the spherical version of both ATLAS9 and ATLAS12, respectively. No extensive grids of models have been published until now, either with ATLAS12, or with any of the two versions of SATLAS.

Instead, extensive grids of ATLAS9 ODF model atmospheres for several metallicities were calculated by Castelli \& Kurucz (2003). These grids are based on solar (or scaled solar) abundances from Grevesse \& Sauval (1998). Recently, Kirby (2011) provided a new ATLAS9 grid, but he used abundances from Anders \& Grevesse (1989). The calculations presented in this paper are based on the more recent solar composition from Asplund et al. (2005). This updated abundance table required new ODFs and Rosseland mean opacity calculations as well. Abundances from Asplund et al. (2005) were chosen instead of those from newer studies (Asplund et al. 2009) to match the composition of the MARCS models described below, and those available from the MARCS Web site.

The MARCS model atmospheres (Gustafsson et al. 1975; Plez et al. 1992; Gustafsson et al. 2008) were developed and have been evolving in close connection with applications primarily 


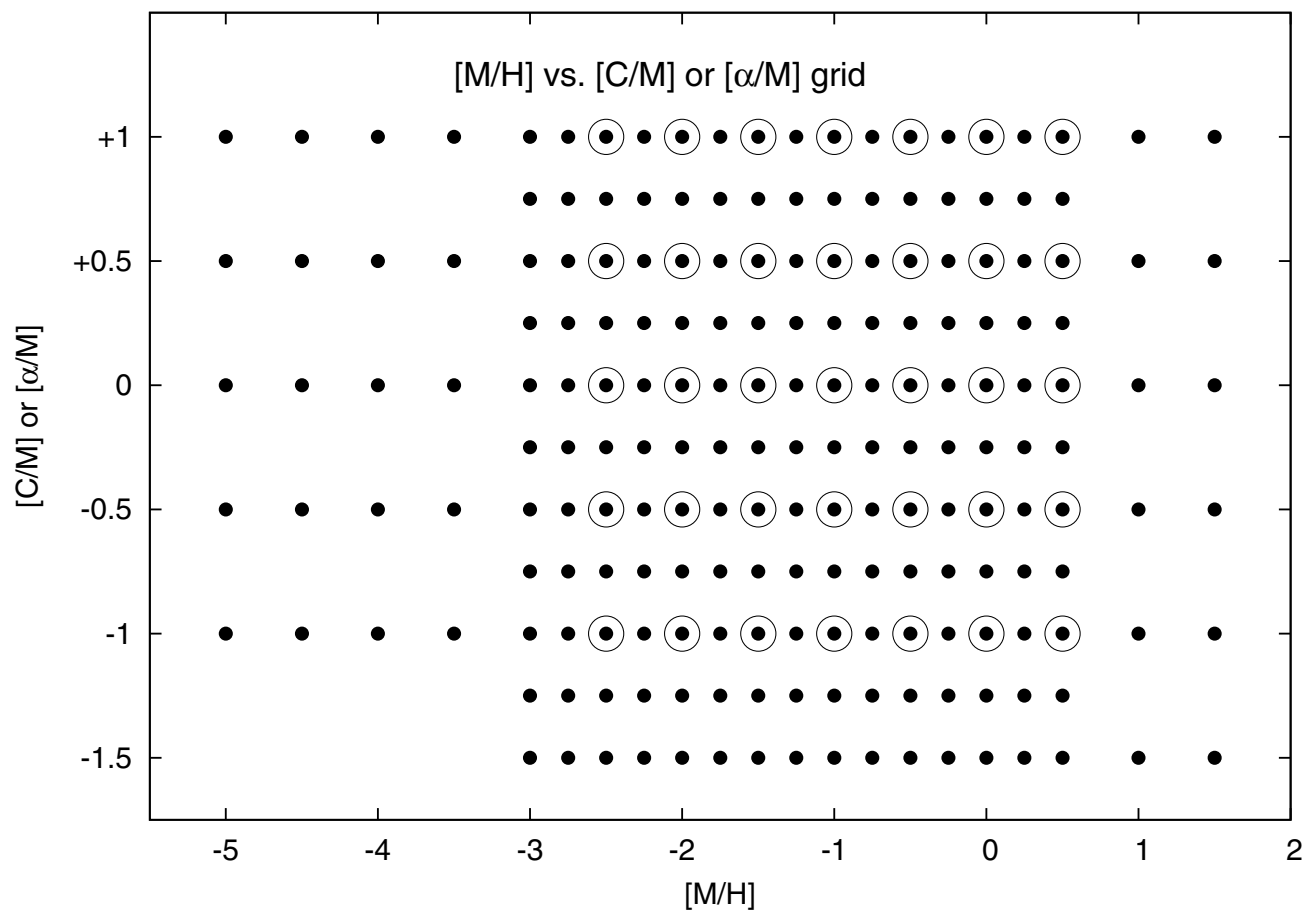

Figure 1. $[\mathrm{C} / \mathrm{M}]$ or $[\alpha / \mathrm{M}]$ content as a function of $[\mathrm{M} / \mathrm{H}]$ of ATLAS9 models (filled circles; Table 1) and MARCS models (open circles; Table 4). Both $[\mathrm{C} / \mathrm{M}]$ and $[\alpha / \mathrm{M}]$ change independently of each other, and the small steps in metallicities give altogether 1980 different compositions for the ATLAS9 models and 175 compositions for the MARCS models. The number of acceptable models may vary for each composition; for details see the ATLAS-APOGEE Web site (http://www.iac.es/proyecto/ATLAS-APOGEE/). For missing metal-rich compositions of ATLAS9 models see Table 2.

to spectroscopic analyses of a wide range of late-type stars with different properties. The models are one-dimensional planeparallel or spherical, and computed in LTE assuming the mixinglength scheme for convective energy transport, as formulated by Henyey et al. (1965). For luminous stars (giants), where the geometric depth of the photosphere is a non-negligible fraction of the stellar radius, the effects of the radial dilution of the energy transport and the depth-varying gravitational field is taken into account. Initially, spectral line opacities were economically treated by the ODF approximation, but later the more flexible and realistic OS scheme has been adopted. In the OS scheme, line opacities are directly tabulated for a large number of wavelength points $\left(10^{5}\right)$ as a function of temperature and pressure.

The shift in the MARCS code from using ODFs to the OS scheme avoided the sometimes unrealistic assumption that the line opacities of certain relative strengths within each ODF wavelength interval overlap in wavelength irrespective of depth in the stellar atmosphere. This assumption was found to lead to systematically erroneous models, in particular when polyatomic molecules add important opacities to surface layers (Ekberg et al. 1986). The current version of the MARCS code used for the present project and for the more extensive MARCS model atmosphere database ${ }^{10}$ was presented and described in detail by Gustafsson et al. (2008). The model atmospheres presented in this paper add large variety in $[\mathrm{C} / \mathrm{M}]$ and $[\alpha / \mathrm{M}]$ abundances to the already existing grids by covering these abundances systematically from -1 to +1 for each metallicity.

Our main purpose is to update the previous ATLAS9 grid and publish new MARCS models to provide a large composition range to use in the APOGEE survey and future precise abundance analysis projects. These new ATLAS models were calculated with a corrected $\mathrm{H}_{2} \mathrm{O}$ line list. The abundances used for the

\footnotetext{
10 http://marcs.astro.uu.se/
}

MARCS models presented in this paper are from Grevesse et al. (2007), which are nearly identical to Asplund et al. (2005); the only significant difference is an abundance of scandium $0.12 \mathrm{dex}$ higher than in Asplund et al. (2005). The range of stellar parameters $\left(T_{\text {eff }}, \log g\right.$ and $\left.[\mathrm{M} / \mathrm{H}]\right)$ spanned by the models covers most stellar types found in the Milky Way.

This paper is organized as follows. In Section 2, we describe the parameter range of our ODFs and model atmospheres and give details of the calculation method of ATLAS9 which we implemented. Section 3 contains the parameter range and calculation procedure for MARCS models. In Section 4, we compare the MARCS and ATLAS9 models with Castelli \& Kurucz (2003), and illustrate how different $\mathrm{C}$ and $\alpha$ contents affect the atmosphere. Section 5 contains the conclusions. The grid of ODFs and model atmospheres will be periodically updated in the future and available online. ${ }^{11}$

\section{ATLAS9 MODEL ATMOSPHERES}

\subsection{Parameters}

The metallicity $([\mathrm{M} / \mathrm{H}])$ of the grid varies from -5 to 1.5 to cover the full range of chemical compositions and is scaled to solar abundances. ${ }^{12}$ For each of these solar scaled compositions we also vary the $[\mathrm{C} / \mathrm{M}]$ and $[\alpha / \mathrm{M}]$ abundances from -1.5 to 1 (Figure 1). ODFs and Rosseland opacity files were calculated with microturbulent velocities $v_{\mathrm{t}}=0,1,2,4,8 \mathrm{~km} \mathrm{~s}^{-1}$, while the model atmospheres were produced only with $v_{\mathrm{t}}=$ $2 \mathrm{~km} \mathrm{~s}^{-1}$. The metallicity grids were the same for all effective temperatures, and the range can be seen in Table 1. Some metalrich compositions with high $\mathrm{C}$ but low $\alpha$ content were not

\footnotetext{
11 http://www.iac.es/proyecto/ATLAS-APOGEE/

$12[\mathrm{M} / \mathrm{H}]$ means any element with $Z>2$ and $[\mathrm{M} / \mathrm{H}]=$ $\log _{10}\left(N_{\mathrm{M}} / N_{\mathrm{H}}\right)_{\star}-\log _{10}\left(N_{\mathrm{M}} / N_{\mathrm{H}}\right)_{\odot}$, where $N_{\mathrm{Fe}}$ and $N_{\mathrm{H}}$ are the number of the desired element and hydrogen nuclei per unit volume, respectively.
} 

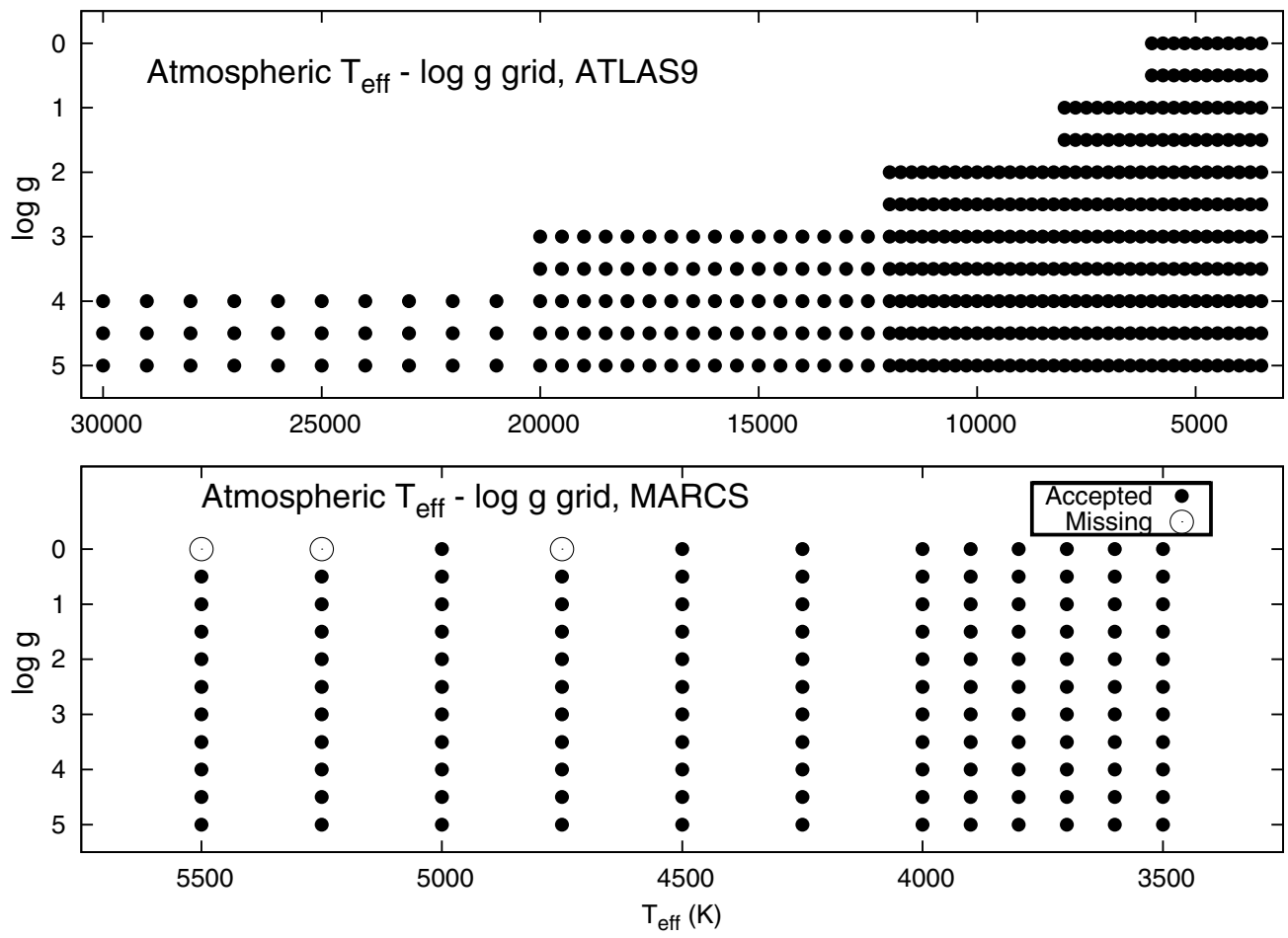

Figure 2. Gravity $\left(\log g\right.$ ) as a function of effective temperature ( $\left.T_{\text {eff }}\right)$ of ATLAS9 and MARCS models calculated for each composition. Acceptable models are denoted by filled circles, while models not acceptable (and missing) are denoted by open circles for the solar composition. Models with $\mathrm{C} / \mathrm{O}>1.7$ and $T_{\text {eff }}<4000 \mathrm{~K}$ are not published (see Section 4.2).

Table 1

Abundance Parameters of ATLAS9 Models

\begin{tabular}{llll}
\hline \hline Parameter & Min & Max & Step \\
\hline$[\mathrm{M} / \mathrm{H}]$ & -5 & -3.5 & 0.5 \\
{$[\mathrm{C} / \mathrm{M}]$} & -1 & 1 & 0.5 \\
{$[\alpha / \mathrm{M}]$} & -1 & 1 & 0.5 \\
\hline$[\mathrm{M} / \mathrm{H}]$ & -3 & 0.5 & 0.25 \\
{$[\mathrm{C} / \mathrm{M}]$} & -1.5 & 1 & 0.25 \\
{$[\alpha / \mathrm{M}]$} & -1.5 & 1 & 0.25 \\
\hline$[\mathrm{M} / \mathrm{H}]$ & 1 & 1.5 & 0.5 \\
{$[\mathrm{C} / \mathrm{M}]$} & -1.5 & 1 & 0.5 \\
{$[\alpha / \mathrm{M}]$} & -1.5 & 1 & 0.5 \\
\hline
\end{tabular}

Table 2

Missing Compositions of ATLAS9 Models

\begin{tabular}{lcc}
\hline \hline$[\mathrm{M} / \mathrm{H}]$ & {$[\mathrm{C} / \mathrm{M}]$} & {$[\alpha / \mathrm{M}]$} \\
\hline 1 & 1 & -1.5 \\
1 & 1 & -1 \\
1.5 & 0.5 & -1.5 \\
1.5 & 1 & -1.5 \\
1.5 & 1 & -1 \\
1.5 & 1 & -0.5 \\
1.5 & 1 & 0 \\
\hline
\end{tabular}

calculated due to excessive computation time; these are listed in Table 2 . The $\alpha$ elements considered when varying $[\alpha / \mathrm{M}]$ were the following: $\mathrm{O}, \mathrm{Ne}, \mathrm{Mg}, \mathrm{Si}, \mathrm{S}, \mathrm{Ca}$, and Ti. The temperature and gravity parameter grid for each composition and spectral type is given in Table 3 . The $T_{\text {eff }}-\log g$ distribution is plotted in Figure 2. Extreme metal-poor and metal-rich compositions were also included.

All the ATLAS codes use atomic and molecular line lists made available by Kurucz on a series of CDROMs. They can
Table 3

Model Atmosphere Parameters of ATLAS9 Models

\begin{tabular}{lrrrccc}
\hline \hline Spectral Type & $T_{\min }$ & $T_{\max }$ & $T_{\text {step }}$ & $\log g_{\min }$ & $\log g_{\max }$ & $\log g_{\text {step }}$ \\
\hline M, N, R, K, G & 3500 & 6000 & 250 & 0 & 5 & 0.5 \\
F & 6250 & 8000 & 250 & 1 & 5 & 0.5 \\
A & 8250 & 12000 & 250 & 2 & 5 & 0.5 \\
B & 12500 & 20000 & 500 & 3 & 5 & 0.5 \\
B, O & 21000 & 30000 & 1000 & 4 & 5 & 0.5
\end{tabular}

now be found at Kurucz's Web site. ${ }^{13}$ The molecular line lists for $\mathrm{TiO}$ and $\mathrm{H}_{2} \mathrm{O}$ were provided by Schwenke (1998) and Partridge \& Schwenke (1997), respectively, and reformatted by Kurucz in ATLAS format. These are also available for download at Kurucz's Web site. For these models, we used the same line lists as Castelli \& Kurucz (2003), except for $\mathrm{H}_{2} \mathrm{O}$, for which a new Kurucz release of the Partridge \& Schwenke (1997) data was adopted. $^{14}$

The solar reference abundance table was adopted from Asplund et al. (2005). Convection was turned on with the mixing-length parameter set to $l / H_{p}=1.25$, but the convective overshooting was turned off. All the models have the same 72 layers from $\log \tau_{\text {Ross }}=-6.875$ to 2 , where the step is $\log$ $\tau_{\text {Ross }}=0.125$. These parameters remained the same as Castelli \& Kurucz (2003) for easy comparison.

All computations were performed on the Diodo cluster at the Instituto Astrofisico de Canarias. Diodo consists of 1 master node and 19 compute nodes, for a total of 80 cores and $256 \mathrm{~GB}$ of RAM, communicating through two independent Gigabit Ethernet networks. Sixteen of the compute nodes host 2 Intel Xeon $3.20 \mathrm{GHz}$ EM64T processors each, with $4 \mathrm{~GB}$ of RAM

\footnotetext{
$13 \mathrm{http}: / /$ kurucz.harvard.edu/linelists.html

14 http://kurucz.harvard.edu/MOLECULES/H2O/h2ofastfix.bin
} 
Table 4

Abundance Parameters of MARCS Models

\begin{tabular}{lllr}
\hline \hline Parameter & Min & Max & Step \\
\hline$[\mathrm{M} / \mathrm{H}]$ & -2.5 & 0.5 & 0.5 \\
{$[\mathrm{C} / \mathrm{M}]$} & -1 & 1 & 0.5 \\
{$[\alpha / \mathrm{M}]$} & -1 & 1 & 0.5 \\
\hline
\end{tabular}

(2 GB per core); the remaining 3 compute nodes each host 16 Intel Xeon (E7340) $2.40 \mathrm{GHz}$ EM64T processors, with $64 \mathrm{~GB}$ of RAM (4 GB per core). On this cluster, about three months of computer time was required for ODF and model atmosphere calculations, using all 80 processors.

\subsection{Calculation Method}

Two separate scripts were developed, one for the ODF and Rosseland opacity calculations, and one for the ATLAS9 calculations. The ODF and Rosseland opacity calculations followed exactly the procedure described by Castelli \& Kurucz (2003), and Castelli (2005b) using the DFSYNTHE code for the ODF, KAPPA9 code for the Rosseland opacity, and ATLAS9 for the model atmosphere calculations (Sbordone 2004, 2005). These codes were compiled in Linux with the Intel Fortran compiler version 11.1.

Our algorithm sets up the initial starting models from the grid provided by Castelli \& Kurucz (2003). ${ }^{15}$ The algorithm chooses the model that has the closest composition, effective temperature, and $\log g$ to the desired output, and an initial ATLAS9 model is calculated. The result must be checked to see whether the output model satisfies the convergence parameters provided by the user for each layer in the model atmosphere. These parameters were set to $1 \%$ for the flux or $10 \%$ for the flux derivative errors after 30 iterations in each run, as recommended in the ATLAS cookbook. ${ }^{16}$ A model is considered converged if the convergence parameters satisfy these criteria in all depths.

We then determined that an atmospheric model is acceptable if one of the following criteria is satisfied: (1) the model has converged through the whole atmosphere, (2) no more than one non-converged layer exists between $\log \tau_{\text {Ross }}=-4$ and $\log$ $\tau_{\text {Ross }}=1$. The model is allowed to have other non-converged layers for $\log \tau_{\text {Ross }}<-4$. A model was considered unacceptable in all other cases. We used only $\log \tau_{\text {Ross }} \geqslant-4$ to $\log$ $\tau_{\text {Ross }}=1$ to check the convergence because most of the lines in the optical and $H$ bands form in this region. In case the output was not acceptable, we restarted the calculation using more iterations. In case of a run with unacceptable output, we selected a starting model that had a different $\log g$ from the initial starting model and used it to restart the calculation. Then the previously described convergence test was performed and more restarts were done if it was necessary. If the output remained unacceptable, the effective temperature of the output model was changed by 10,50 , and $100 \mathrm{~K}$. If the output of any of these runs was acceptable, then it was used as an input model to calculate the atmosphere with the original effective temperature.

To test our model atmospheres, we replicated the Rosseland opacity calculations by Castelli \& Kurucz (2003). For this we used Castelli's scripts without any modification to calculate the ODF and Rosseland opacities for the Grevesse \& Sauval (1998) abundances. These calculations concluded in perfect agreement within numerical precision. We then attempted to reproduce

\footnotetext{
15 http://wwwuser.oat.ts.astro.it/castelli/

16 http://atmos.obspm.fr/index.php/documentation
}

Table 5

Model Atmosphere Parameters of MARCS Models

\begin{tabular}{|c|c|c|c|c|c|c|}
\hline Spectral Type & $T_{\min }$ & $T_{\max }$ & $T_{\text {step }}$ & $\log g_{\min }$ & $\log g_{\max }$ & $\log g_{\text {step }}$ \\
\hline $\mathrm{M}, \mathrm{N}, \mathrm{R}, \mathrm{K}^{\mathrm{a}}$ & 3500 & 4000 & 100 & 0 & 3 & 0.5 \\
\hline $\mathrm{K}, \mathrm{G}^{\mathrm{a}}$ & 4250 & 5500 & 250 & 0 & 3 & 0.5 \\
\hline $\mathrm{M}, \mathrm{N}, \mathrm{R}, \mathrm{K}^{\mathrm{b}}$ & 3500 & 4000 & 100 & 3.5 & 5 & 0.5 \\
\hline $\mathrm{K}, \mathrm{G}^{\mathrm{b}}$ & 4250 & 5500 & 250 & 3.5 & 5 & 0.5 \\
\hline
\end{tabular}

Notes.

a Spherical atmospheres.

b Plane-parallel atmospheres.

model atmospheres with the same parameters found on Castelli's Web site with our scripts using the ODFs and Rosseland opacity files generated with Grevesse \& Sauval (1998) abundances. This test also concluded with near perfect agreement with only $0.1-0.2 \mathrm{~K}$ maximum differences coming from the different version of compilers used.

\section{MARCS MODEL ATMOSPHERES}

\subsection{Parameters}

Models were computed for seven overall metallicities, $[\mathrm{M} / \mathrm{H}]$ from -2.5 to 0.5 , with a step size of $0.5 \mathrm{dex}$. For each of these seven overall $[\mathrm{M} / \mathrm{H}]$ mixtures, 25 combinations of modified carbon and $\alpha$-element abundances were adopted: the modifications to the logarithmic $\mathrm{C}$ and $\alpha$ abundances are $-1,-0.5$, $0,0.5$, and 1 dex. This format resulted in a total of 175 subgrids with unique chemical compositions (Table 4). The $\alpha$ elements in MARCS are O, Ne, Mg, Si, S, Ar, Ca, and Ti. This composition scheme is exactly the same as the previous models found on the MARCS Web site. The systematic $\alpha$ abundance changes were chosen to overlap the scheme (Section 2.1) used in the ATLAS9 model calculations.

For each of these abundance subgrids, models with 12 values of effective temperature from 3500 to $5500 \mathrm{~K}$ and 11 values of logarithmic surface gravities from 0 to 5 were computed (see Figure 2 and Table 5). Models with logarithmic surface gravities lower than 3.5 (giants) were computed in spherical geometry and with a microturbulence parameter of $2 \mathrm{~km} \mathrm{~s}^{-1}$, while the remaining (dwarf) models adopted $v_{\mathrm{t}}=1 \mathrm{~km} \mathrm{~s}^{-1}$ and plane-parallel geometry. In the end, $86 \%$ of the 23,140 models converged satisfactorily. Convergence was particularly poor for cool dwarfs that are simultaneously $\alpha$-rich and carbon-poor.

Details about the atomic and molecular line lists used by the MARCS code are given by Gustafsson et al. (2008). In a number of instances, they are different from those used by the ATLAS code, as for $\mathrm{H}_{2} \mathrm{O}$ (Barber et al. 2006), and TiO (Plez 1998).

\subsection{Calculation Method}

Similar to the ATLAS9 ODF calculations, a new metallicity MARCS subgrid is started with the summation of an OS table of atomic line opacities for the relevant abundance mixture and microturbulence parameter. Since line opacity data are needed for many atoms and first ions, it saves time to add the opacities of the individual species into one file before a set of models is computed. This file contains a table with over $10^{5}$ wavelength points with line opacities relevant to the equation of state for 306 combinations of temperature and damping pressure $P_{6}$. The damping pressure is used as a proxy for the pressure that broadens metal lines by collisions with neutral atoms in cool stars. $P_{6}$ is the pressure of $\mathrm{H}_{\mathrm{I}}$ with the addition of the 
polarizability corrected pressures of neutral helium and $\mathrm{H}_{2}$ (see Equation (33) of Gustafsson et al. 2008). For each molecule, in contrast, the line opacities are given in one table for the same wavelength set, but as a function of temperature and microturbulence parameter.

The method used for the MARCS model calculations is described in detail in Gustafsson et al. (2008). They start with the generation of a simplified starting model assuming a gray opacity. Physical parameters and their derivatives are computed and the model structure is iterated in a multidimensional Newton-Raphson scheme until the flux through each depth layer corresponds to the prescribed effective temperature. The models usually converged after $4-8$ iterations; convergence also requires that the maximum temperature correction in any depth point is below $1.5 \mathrm{~K}$ during two consecutive iterations. Occasionally convergence takes longer, and some models do not converge at all. A converged model with similar model parameters is then identified as a new starting model. This approach is often successful, but some models do not converge, which leaves vacancies in the model grid.

\section{DISCUSSION}

Changes in the chemical composition can have a number of effects on the calculated atmospheres. These effects are mainly related to either changes in opacity or changes in the equation of state. The main effect of an increased line opacity in late-type stars is either cooling or warming (depending on the opacity and its wavelength dependence) of the outer layers and also back-warming of the innermost ones (Gustafsson et al. 1975). Changes in the equation of state are mainly variations in the mean molecular weight for abundant elements, changes in the number of free electrons for elements that are important electron donors, or other more intricate changes related to chemical equilibrium through molecule formation. In the next two sections, we give examples of MARCS and ATLAS9 models from our grid, and illustrate briefly the changes in the model atmospheres related to large changes in $\mathrm{C}$ and $\alpha$ elements.

\subsection{Comparing our MARCS and ATLAS9 Models to the Castelli-Kurucz Grid}

Figure 3 illustrates examples of the atmospheric structures for two models with solar composition, one for $T_{\text {eff }}=4000 \mathrm{~K}$ and $\log g=1$ (red line) and the other for $T_{\text {eff }}=5500 \mathrm{~K}$ and $\log g=4$ (blue line). There are four double panels showing the parameter dependence with Rosseland optical depth of the mass column (top-left panel), temperature (top-right), gas pressure (bottom-left), and electron number density (bottom-right). The dashed, solid, and dotted lines correspond to the new APOGEE MARCS, APOGEE Kurucz, and earlier (NEWODF) Castelli-Kurucz models (Castelli \& Kurucz 2003), respectively. While the APOGEE Kurucz and MARCS models share essentially the same chemical composition (Asplund et al. 2005; Grevesse et al. 2007), the Castelli-Kurucz models use the solar mixture given by Grevesse \& Sauval (1998).

For both the cooler (red line) and the warmer model (blue line), we see good agreement between the MARCS and the ATLAS9 models presented in this paper. The new MARCS and ATLAS9 models show good agreement, the differences are modest, less than $1 \%$, for the thermal structure, and less than $2 \%-3 \%$ for the gas pressure and the electron density in the layers where weak spectral lines and continuum form $(\tau>0.01)$. These small differences are most likely related to
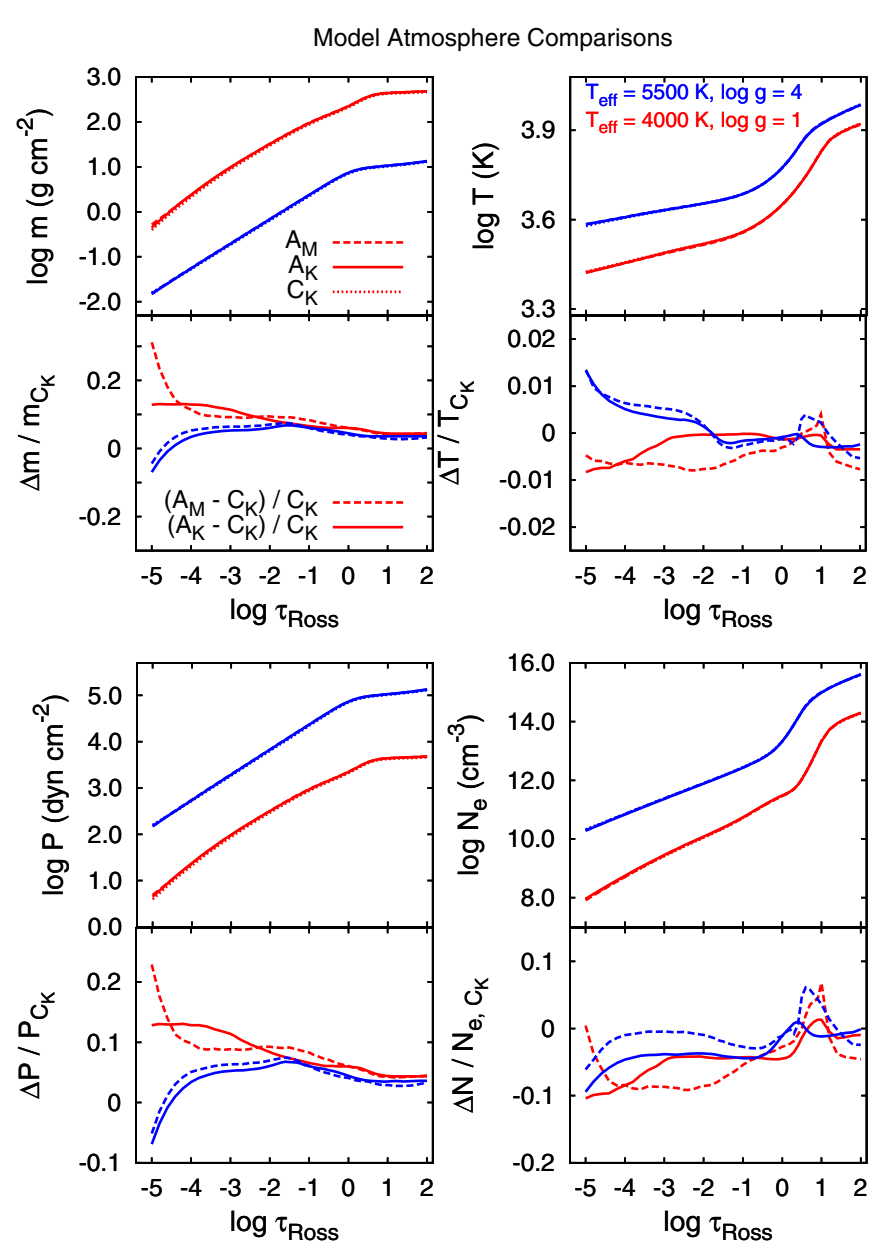

Figure 3. Examples of ATLAS9 and MARCS model atmospheres with $T_{\text {eff }}=$ $4000 \mathrm{~K}$ and $\log g=1$ (red line in the online version) and $T_{\text {eff }}=5500 \mathrm{~K}$ and $\log g=4$ (blue line in the online version). The model from the APOGEE grid is denoted by a solid line, the corresponding ATLAS9 model from Castelli \& Kurucz (2003) is denoted by a dotted line, and the MARCS model is denoted by a dashed line. The panels show the mass column $(m)$, temperature $(T)$, gas pressure $(P)$, electron number density $\left(N_{\mathrm{e}}\right)$ as a function of the logarithm of optical depth $(\log \tau)$, and the relative difference between the ATLAS9 $\left(A_{\mathrm{K}}\right)$, MARCS $\left(A_{\mathrm{M}}\right)$ calculations presented in this paper and Castelli \& Kurucz (2003) $\left(C_{\mathrm{K}}\right)$.

(A color version of this figure is available in the online journal.)

the different equation of state implemented in the two codes, and can also be due to the fact that ATLAS9 uses the ODF method, while MARCS uses the OS method. The differences between the two increase at temperatures lower than $4000 \mathrm{~K}$ due to the different $\mathrm{H}_{2} \mathrm{O}$ and $\mathrm{TiO}$ line lists used in the calculations. More importantly, larger differences are present in the gas pressure and electron density in the ATLAS9 models compared to the Castelli-Kurucz ones for the cooler models. These differences must be related to the updated solar chemical composition, as the Castelli-Kurucz models are also computed with a corrected $\mathrm{H}_{2} \mathrm{O}$ line list. The most significant change in the solar composition corresponds to the reduction in oxygen, nitrogen, and carbon abundances, all of which decrease in the update. This causes a reduction in the Rosseland opacity at a given temperature and gas pressure. The decreased Rosseland opacity leads to a subsequent increase in the total pressure, which is only partially compensated by a reduction in the electron density.

Gustafsson et al. (2008) present some further comparisons between MARCS models and ATLAS9 models of Castelli \& Kurucz (2003). Other comparisons between MARCS, ATLAS9, 


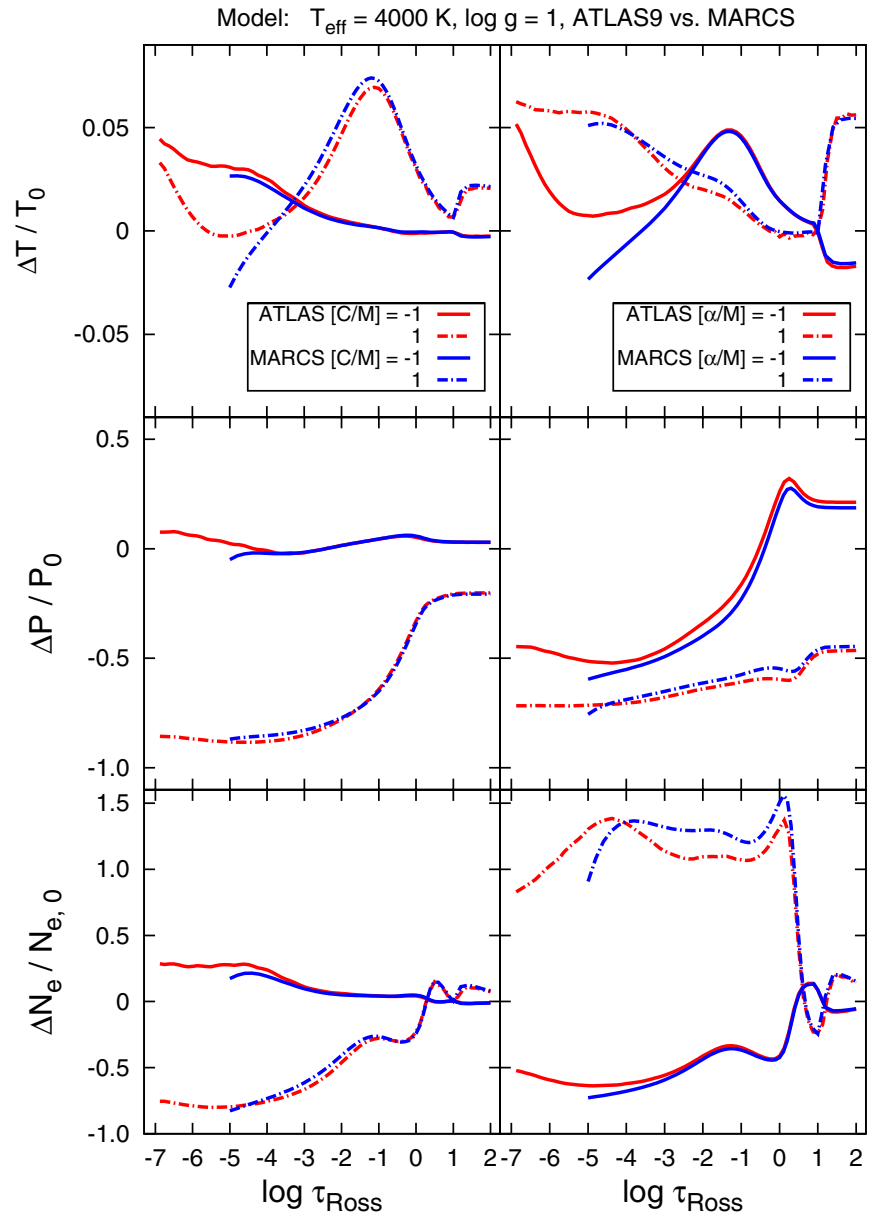

Figure 4. Examples of how different $[\mathrm{C} / \mathrm{M}]$ and $[\alpha / \mathrm{M}]$ content changes the temperature profile in the ATLAS9 and MARCS atmospheres for $T_{\mathrm{eff}}=4000 \mathrm{~K}$, $\log g=1$. This figure shows the temperature $(T)$, gas pressure $(P)$, electron number density $\left(N_{\mathrm{e}}\right)$, and the relative difference (where 0.5 corresponds to $50 \%$, 1 corresponds to $100 \%$ ) of these parameters between $[\mathrm{C} / \mathrm{M}]=[\alpha / \mathrm{M}]=0$ and $[\mathrm{C} / \mathrm{M}]=[\alpha / \mathrm{M}]=-1$, and 1 as a function of optical depth $(\log \tau)$. The symbols $T_{0}, P_{0}, N_{e, 0}$ correspond to $[\mathrm{C} / \mathrm{M}]=[\alpha / \mathrm{M}]=0$.

(A color version of this figure is available in the online journal.)

and PHOENIX model atmospheres and spectra were discussed by Plez (2011).

\subsection{Changes in Model Structures Related to Chemical Composition}

Figures 4 and 5 illustrate the changes in the model structures in response to changes in chemical composition. The left-hand panels of Figures $4\left(T_{\text {eff }}=4000 \mathrm{~K}\right.$ model $)$ and $5\left(T_{\text {eff }}=5500 \mathrm{~K}\right)$ show the relative changes associated with variations of the carbon abundance from solar proportions. The ATLAS9 model for the warmer temperature has $v_{\mathrm{t}}=2 \mathrm{~km} \mathrm{~s}^{-1}$, while the MARCS model has $v_{\mathrm{t}}=1 \mathrm{~km} \mathrm{~s}^{-1}$, but this difference does not affect the model structures significantly. The relative variations found are very similar for MARCS and ATLAS9, except in the outermost layers of the atmosphere. The changes in the thermal structure are modest and show a behavior symmetric to that found for oxygen, which strongly suggests that $\mathrm{CO}$ formation is the driver of the variation. $\mathrm{CO}$ is the most tightly bound molecule and consumes almost all of the free atoms of either carbon or oxygen, whichever is less abundant, leaving the majority species ( $\mathrm{C}_{\mathrm{I}}$ or $\mathrm{O}$ I) to form other molecules. If oxygen dominates, it produces a "normal star" (if cool, an $M$ star), otherwise carbon is free to

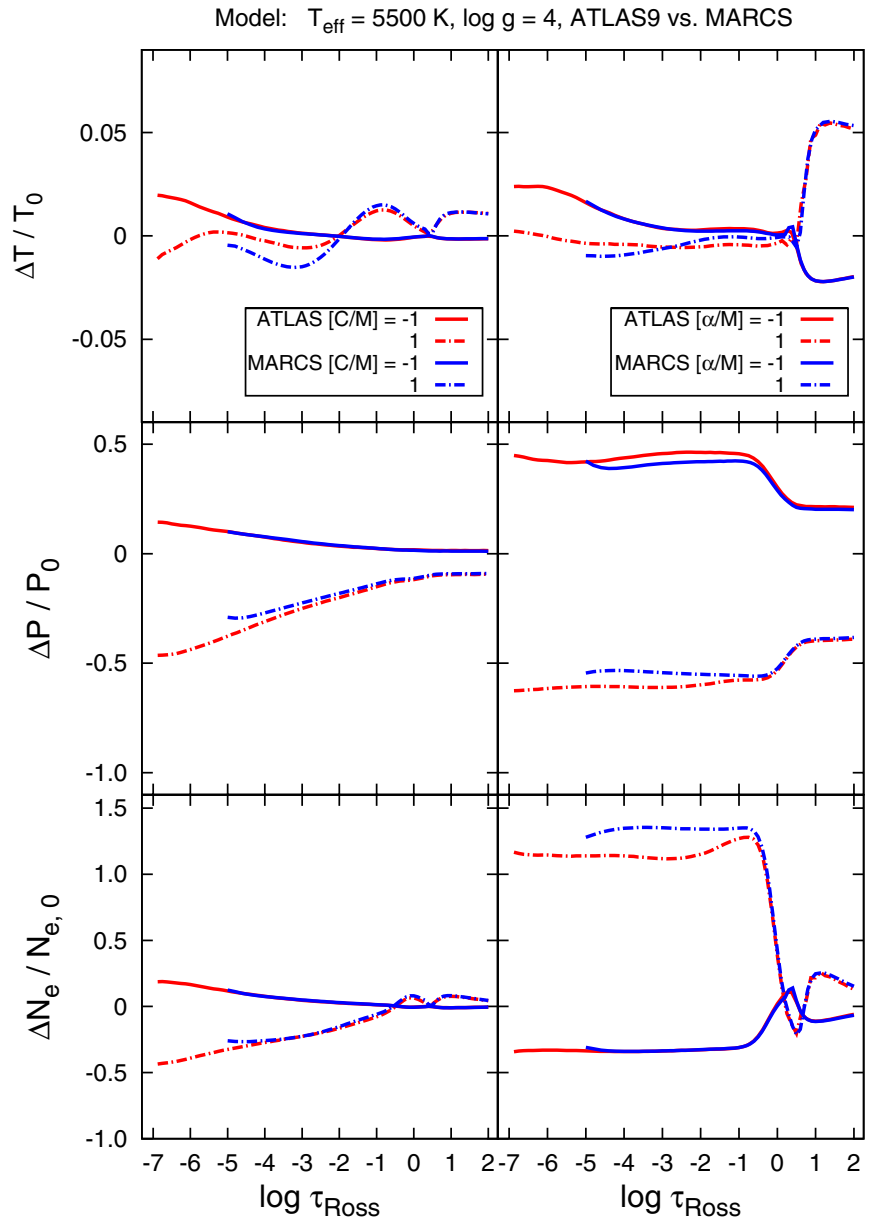

Figure 5. Examples of how different $[\mathrm{C} / \mathrm{M}]$ and $[\alpha / \mathrm{M}]$ content changes the temperature profile in the ATLAS9 and MARCS atmospheres for $T_{\text {eff }}=5500 \mathrm{~K}$, $\log g=4$. For more details see the caption of Figure 6 .

(A color version of this figure is available in the online journal.)

form many molecules with high opacities, making carbon stars with very different spectra. At $T_{\text {eff }}=5500 \mathrm{~K}, \mathrm{CO}$ formation is low, thus this molecule does not affect the chemical equilibrium, contrary to what is seen in the cooler atmospheres.

The right-hand panels show changes for the same model parameters and different $\alpha$-element abundances. Large changes are visible for both models in the pressure and electron numbers. The significant differences in the pressure compared to the cooler model are due to increased gravity. Increasing the abundance of $\alpha$ elements reduces pressure (despite the fact that electron pressure increases) because the electrons of the other main electron contributors change the continuum opacity. The $\alpha$ elements that contribute most to the total number of electrons in the ATLAS9 models are shown in Figure 6. For the $T_{\text {eff }}=4000 \mathrm{~K}, \log g=1$ model, the main electron contributors are $\mathrm{Ca}, \mathrm{Mg}, \mathrm{Na}$, and $\mathrm{Al}$ in the outer layers and $\mathrm{Mg}, \mathrm{Si}$, and $\mathrm{Fe}$ in the deeper layers. For the warmer models, this changes significantly, as more $\mathrm{Fe}$ and $\mathrm{H}$ are ionized, the overall number of electrons increases and the main electron contributors become $\mathrm{Mg}, \mathrm{Si}$, and $\mathrm{Fe}$ through most of the atmospheres.

Internal tests showed that polyatomic carbon molecules $\left(\mathrm{C}_{2} \mathrm{H}_{2}, \mathrm{C} 3\right)$ substantially change the structure of the atmosphere with $\mathrm{C} / \mathrm{O}$ ratios higher than 1.7, if they are included in the line list. These molecules significantly inflate the atmosphere changing the temperature in the line-forming photospheric 


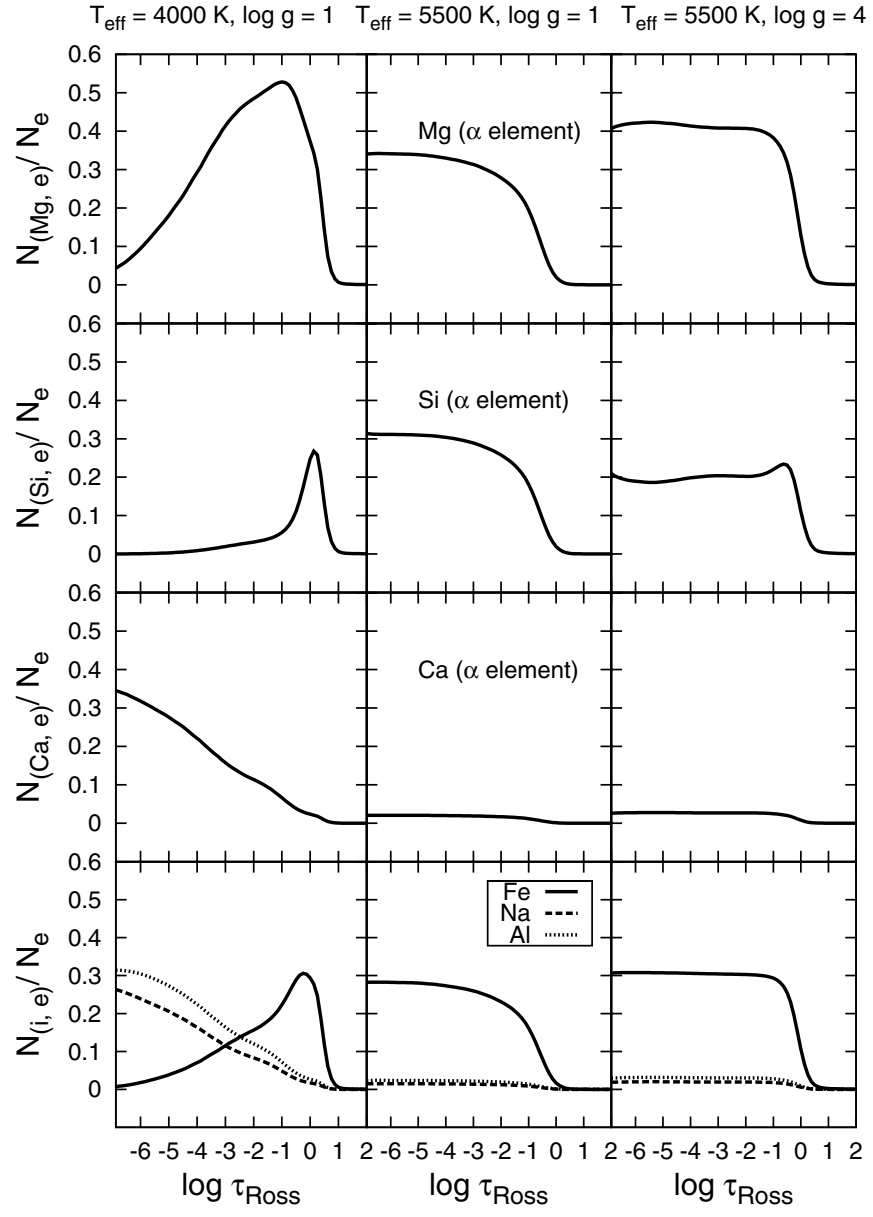

Figure 6. Examples of the largest electron contributors to the total electron numbers in the ATLAS9 atmospheres for $[\mathrm{M} / \mathrm{H}]=[\mathrm{C} / \mathrm{M}]=[\alpha / \mathrm{M}]=0$. The figure shows the element electron numbers relative to the total number of electrons for the two models used in previous figures. The $T_{\text {eff }}=5500 \mathrm{~K}$ $\log g=1$ model is plotted in the middle section to show that the electron contributors do not change significantly compared to a model with the same temperature but higher gravity.

layers. Since these molecules were used neither in the ATLAS9, nor in the MARCS calculations, atmospheric structures with $\mathrm{C} / \mathrm{O}>1.7$ and $T_{\text {eff }}<4000 \mathrm{~K}$ are not reliable and thus not published.

\section{CONCLUSIONS}

Most of the ATLAS9 models are fully converged above $T_{\text {eff }}=$ $5000 \mathrm{~K}$. Convergence problems are visible only in the outermost layers for stars with $T_{\text {eff }}<5000 \mathrm{~K}$. The regions affected by convergence issues are limited to $\log \tau_{\text {Ross }}<-4$. However, these unconverged layers on the top of the atmosphere at low temperatures do not affect most line profiles very significantly because most lines form deeper in the atmosphere. A little over a million ATLAS9 atmospheres were calculated.

Over 20,000 MARCS fully converged models with ranges in $[\mathrm{C} / \mathrm{M}]$ and $[\alpha / \mathrm{M}]$ have been produced. Models are available in spherical and plane-parallel cases. Convergence issues are also present for red dwarf stars, similar to the ATLAS9 models.

Examples were presented for both ATLAS9 and MARCS models for $T_{\text {eff }}=4000 \mathrm{~K}$ and $5500 \mathrm{~K}$, and compared to the Castelli-Kurucz grid. The new ATLAS9 and MARCS models agree well for all temperatures, while the differences between these calculations and the Castelli-Kurucz grid arise from the updated abundance tables. We briefly illustrated the effects of decreased/increased carbon and $\alpha$ content on the structure of the atmospheres, which are very similar in the MARCS and ATLAS9 models. The response to the carbon content is only different in the outermost layers due to increased $\mathrm{CO}$ in the atmosphere. The response to $\alpha$ elements is also almost the same in MARCS and ATLAS9 models. The increased $\alpha$-element content has profound effects on electron numbers and pressure for both the giant and dwarf stars, which is related to the higher number of $\mathrm{Mg}, \mathrm{Si}$, and $\mathrm{Ca}$ ions. Carbon-rich models with $\mathrm{C} / \mathrm{O}>1.7$ and $T_{\text {eff }}<4000 \mathrm{~K}$ are not published here because polyatomic carbon molecules not included in the line lists here significantly change the temperature structure in the photosphere.

These model grids will be used as the primary database for the pipeline analyzing the spectra from the APOGEE survey. The high-resolution model spectra used in the survey's atmospheric parameters and abundances determination code will be built on the models presented in this paper. Both the ATLAS9 and MARCS models' atmospheres will be continuously updated with new compositions as the APOGEE survey progresses. The calculated ODFs, Rosseland opacities available for $v_{\mathrm{t}}=0,1$, $2,4,8 \mathrm{~km} \mathrm{~s}^{-1}$, and the ATLAS9 model atmosphere files are available for $v_{\mathrm{t}}=2 \mathrm{~km} \mathrm{~s}^{-1}$ from the ATLAS-APOGEE Web site. ${ }^{17}$ The MARCS models are available with $v_{\mathrm{t}}=2 \mathrm{~km} \mathrm{~s}^{-1}$ for the giant and $v_{\mathrm{t}}=1 \mathrm{~km} \mathrm{~s}^{-1}$ for the dwarf stars from the standard MARCS Web site. ${ }^{18}$

We are grateful to Robert L. Kurucz, who kindly provided us with the new and corrected $\mathrm{H}_{2} \mathrm{O}$ line list. We thank Katia Cunha and Verne Smith for helping us with the discussion of Kurucz and MARCS models. We also thank Martin Asplund and Kjell Eriksson for their contributions to the MARCS models presented in this paper. Don P. Schneider, Kaike Pan, and David H. Weinberg also greatly improved our work by providing many useful comments and suggestions. Funding for SDSS-III has been provided by the Alfred P. Sloan Foundation, the Participating Institutions, the National Science Foundation, and the U.S. Department of Energy Office of Science. The SDSS-III Web site is http://www.sdss3.org/. SDSS-III is managed by the Astrophysical Research Consortium for the Participating Institutions of the SDSS-III Collaboration including the University of Arizona, the Brazilian Participation Group, Brookhaven National Laboratory, University of Cambridge, Carnegie Mellon University, University of Florida, the French Participation Group, the German Participation Group, Harvard University, the Instituto de Astrofisica de Canarias, the Michigan State/Notre Dame/ JINA Participation Group, Johns Hopkins University, Lawrence Berkeley National Laboratory, Max Planck Institute for Astrophysics, New Mexico State University, New York University, Ohio State University, Pennsylvania State University, University of Portsmouth, Princeton University, the Spanish Participation Group, University of Tokyo, University of Utah, Vanderbilt University, University of Virginia, University of Washington, and Yale University.

\section{REFERENCES}

Aihara, H., Allende Prieto, C., An, D., et al. 2011, ApJS, 193, 29

Allende Prieto, C., Majewski, S. R., Schiavon, R., et al. 2008, Astron. Nachr., 329,1018

\footnotetext{
17 http://www.iac.es/proyecto/ATLAS-APOGEE/

$18 \mathrm{http}: / /$ marcs.astro.uu.se/
} 
Anders, E., \& Grevesse, N. 1989, Geochim. Cosmochim. Acta, 53, 197

Asplund, M., Grevesse, N., \& Sauval, A. J. 2005, in ASP Conf. Ser. 336, Cosmic Abundances as Records of Stellar Evolution and Nucleosynthesis, ed. T. G.

Barnes, III \& F. N. Bash (San Francisco, CA: ASP), 25

Asplund, M., Grevesse, N., Sauval, A. J., \& Scott, P. 2009, ARA\&A, 47, 481

Barber, R. J., Tennyson, J., Harris, G. J., \& Tolchenov, R. N. 2006, MNRAS, 369, 1087

Castelli, F. 2005a, Mem. Soc. Astron. Ital. Suppl., 8, 25

Castelli, F. 2005b, Mem. Soc. Astron. Ital. Suppl., 8, 34

Castelli, F., \& Kurucz, R. L. 2003, in IAU Symp. 210, New Grids of ATLAS9 Model Atmospheres, ed. N. Piskunov, W. W. Weiss, \& D. F. Gray (Cambridge: Cambridge Univ. Press), 20

Eisenstein, D. J., Weinberg, D. H., Agol, E., et al. 2011, AJ, 142, 72

Ekberg, U., Eriksson, K., \& Gustafsson, B. 1986, A\&A, 167, 304

Grevesse, N., Asplund, M., \& Sauval, A. J. 2007, Space Sci. Rev., 130, 105

Grevesse, N., \& Sauval, A. J. 1998, Space Sci. Rev., 85, 161

Gunn, J. E., Siegmund, W. A., Mannery, E. J., et al. 2006, AJ, 131, 2332

Gustafsson, B., Bell, R. A., Eriksson, K., \& Nordlund, Å. 1975, A\&A, 42, 407

Gustafsson, B., Edvardsson, B., Eriksson, K., et al. 2008, A\&A, 486, 951
Henyey, L., Vardya, M. S., \& Bodenheimer, P. 1965, ApJ, 142, 841

Kirby, E. N. 2011, PASP, 123, 531

Kurucz, R. L. 1979, ApJS, 40, 1

Kurucz, R. L. 1993, ATLAS9 Stellar Atmosphere Programs and $2 \mathrm{~km} \mathrm{~s}^{-1}$ grid, Kurucz CD-ROM No. 13 (Cambridge, MA: Smithsonian Astrophysical Observatory)

Kurucz, R. L. 2005, Mem. Soc. Astron. Ital. Suppl., 8, 14

Kurucz, R. L., \& Avrett, E. H. 1981, Solar Spectrum Synthesis. I. A Sample Atlas from 224 to $300 \mathrm{~nm}$, SAO Special Report 391

Lester, J. B., \& Neilson, H. R. 2008, A\&A, 491, 633

Partridge, H., \& Schwenke, D. W. 1997, J. Chem. Phys., 106, 4618

Plez, B. 1998, A\&A, 337, 495

Plez, B. 2011, J. Phys.: Conf. Ser., 328, 012005

Plez, B., Brett, J. M., \& Nordlund, Å. 1992, A\&A, 256, 551

Sbordone, L. 2005, Mem. Soc. Astron. Ital. Suppl., 8, 61

Sbordone, L., Bonifacio, P., Castelli, F., \& Kurucz, R. L. 2004, Mem. Soc. Astron. Ital. Suppl., 5, 93

Schwenke, D. W. 1998, Faraday Discuss., 109, 321

Strom, S. E., \& Kurucz, R. L. 1966, AJ, 71, 181 